\title{
Changes in the Dusty Environment of the Recurrent Nova RS Ophiuchi
}

\author{
M. T. Rushton ${ }^{1}$, L. A. Helton ${ }^{2}$, B. Kaminsky ${ }^{3}$, C. E. Woodward ${ }^{4}$, \\ Ya. V. Pavlenko ${ }^{3}$, and A. Evans ${ }^{5}$ \\ ${ }^{1}$ Jeremiah Horrocks Institute, University of Central Lancashire \\ Preston, PR1 2HE, UK \\ email: mrushton@uclan.ac.uk \\ ${ }^{2}$ SOFIA, NASA Ames Research Centre \\ MS 211-3, Moffat Field, CA 94035, USA \\ ${ }^{3}$ National Academy of Sciences of Ukraine, \\ 27 Zabolonnoho, Kyiv-127,03680, Ukraine \\ ${ }^{4}$ Dep. of Astromy, University of Minnesota, \\ 116 Church Street, S. R., Minneapolis, MN 55455, USA \\ ${ }^{5}$ Astrophysics Group, Keele University, \\ Keele, Staffordshire, ST5 5BG, UK
}

\begin{abstract}
We present Spitzer spectroscopy of the recurrent nova RS Ophiuchi obtained on several occasions between 2006 and 2009. The spectra show variability in the silicate dust features at $9.7 \mu \mathrm{m}$ and $18 \mu \mathrm{m}$, which form in the wind of the red giant in the system. We fit the spectra with Dusty models and find changes in the dust temperature, due to changes in the luminosity of the central source. The mass-loss rate of the secondary is a few $10^{-7} M_{\odot} \mathrm{yr}^{-1}$, typical of a red giant of its type.
\end{abstract}

Keywords. stars: novae, cataclysmic variables, mass loss, winds, outflows

\section{Introduction}

RS Ophiuchi is a recurrent nova which has shown at least eight outbursts since 1898; the most recent occurred in 2006 (Schaefer 2010). The system consists of a white dwarf in a semi-detached binary with a red giant. The short recurrence interval of recurrent novae is usually attributed to a hot, massive white dwarf and a high accretion rate (Starrfield 2008). A review of recurrent novae is given in Anupama (these proceedings), and the possibility that such systems are the progenitors of Type Ia supernovae is discussed in several other contributions.

Dust emission from the red giant wind has been detected in RS Oph on a number of occasions (Geisel 1970, Schaefer 1986, Rushton et al. 2010). Here we present Spitzer IRS spectroscopy obtained during 2006-2009, which show the silicate features at $9.7 \mu \mathrm{m}$ and $18 \mu \mathrm{m}$. The observations began about seven months after the last outburst, covering the post outburst minimum and the recovery to the quiescent state. Figure 1 shows the times of the observations with respect to the visual light curve of RS Oph.

\section{Results}

The Spitzer IRS spectra of RS Oph are shown in Figure 2. The silicate dust features are clearly variable, appearing strongest in the 2007 Sep data. Here we investigate the behavior of the dust emission using the DUSTY code (Ivezic 1999) to determine the grain 


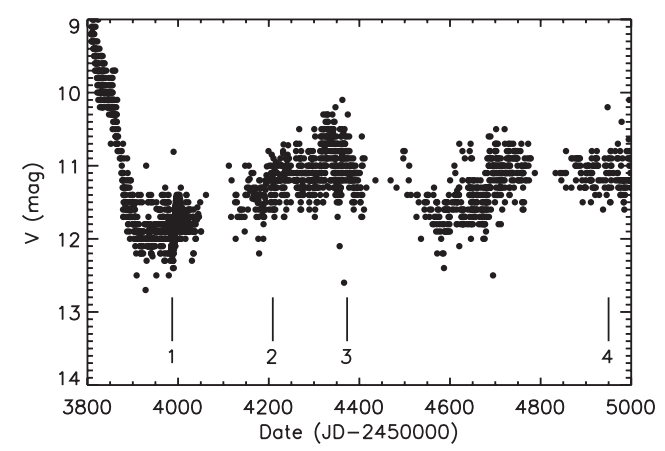

Figure 1. The visual light curve of RS Oph from the American Association of Variable Star Observers (AAVSO) for the period 2006-2009; it shows the decline from the last outburst, the post outburst minimum (about MJD 3900) and the recovery to the quiescent level (about MJD 4400). The vertical lines show the times of the Spitzer observations.

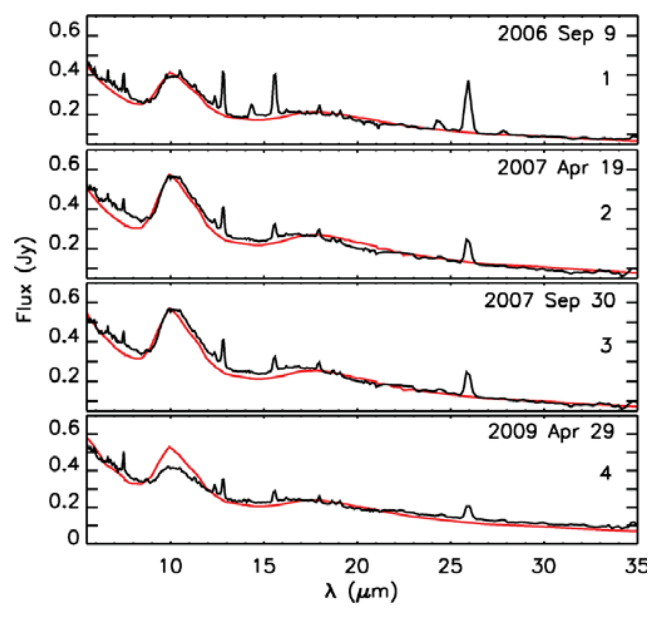

Figure 2. Spitzer IRS spectroscopy of RS Oph on the given dates. The silicate features at $9.7 \mu \mathrm{m}$ and $18 \mu \mathrm{m}$ are visible and variable. The red lines are the best-fitting DUSTY models to the data. The numbers in each panel correspond to those in Figure 1.

parameters. The grain type selected for our models is the warm silicate from Ossenkopf (1992), with a standard MRN size distribution (Mathis 1977). The dust is assumed to be spherically symmetric around the RS Oph system. For the radiation source, we use optical and near infrared fluxes obtained on dates close to the times of the Spitzer observations.

The mass-loss rate of the red giant is found to be a few $10^{-7} M_{\odot} \mathrm{yr}^{-1}$, consistent with several other estimates (Schaefer 2010). We find that an increase in dust temperature, from about $400 \mathrm{~K}$ in $2006 \mathrm{Sep}$ to about $500 \mathrm{~K}$ in $2007 \mathrm{Sep}$, coincides with the recovery from the post outburst minimum, which is usually attributed to the redevelopment of the accretion disk. The rise in temperature suggests that the luminosity of RS Oph increased by a factor of $2-3$; this is consistent with fits to the UVOIR fluxes (Skopal 2008).

The silicate emission is much weaker in the 2009 data and the DusTY fit overestimates the strength of the $9.7 \mu \mathrm{m}$ feature, which depends on the properties of the grains. A thorough analysis of this data will be given elsewhere.

\section{Acknowledgments}

We would like to thank everyone for an enjoyable meeting. We acknowledge with thanks the variable star observations from the AAVSO international database contributed by observers worldwide and used in this research.

\section{References}

Geisel et al. 1970, ApJ, 161, L101

Ivezić et al. 1999, Univ. Kentucky Internal Report

Mathis, J. S. 1977, ApJ, 217, 425

Ossenkopf et al. 1992, A\&SA, 261, 567

Rushton et al. 2010, MNRAS, 401, 99

Schaefer, B. 1986, PASP, 98, 556

Schaefer, B. 2010, ApJs, 187, 75

Skopal et al. 2008, ASP Conf. Ser., 401, 101

Starrfield, S. 2008, ASP Conf. Ser., 401, 4 